Vol. 37
Núm. 78

\title{
Frecuencia de quistes y tumores odontogénicos en población nicaragüense. Estudio retrospectivo a siete años*
}

Frequency of Odontogenic Cysts and Tumors in Nicaraguan Population. A Seven-Year Retrospective Study

\author{
Luis Alberto Quintana Salgado a \\ Universidad Nacional Autónoma de Nicaragua León, \\ Nicaragua \\ luis.quintana@fo.unanleon.edu.ni \\ ORCID: http://orcid.org/0000-0003-4355-6773 \\ Luis Javier Espinoza Hernández \\ Universidad Nacional Autónoma de Nicaragua León, \\ Nicaragua \\ ORCID: http://orcid.org/0000-0002-4696-2403
}

José Javier Rodriguez Vilchez

Práctica privada, Nicaragua

ORCID: http://orcid.org/0000-0001-7318-4881

Carlos David Guerrero Midence

Práctica privada, Nicaragua

ORCID: http://orcid.org/0000-0002-6043-3372

Harley José Rugama Díaz

Práctica privada, Nicaragua

ORCID: http://orcid.org/0000-0002-1532-8119
DOI: https://doi.org/10.11144/Javeriana.uo37-78.fqto Redalyc: http://www.redalyc.org/articulo.oa? $\mathrm{id}=231260072010$

Fecha de recepción: 09 Noviembre 2017 Fecha de aprobación: 16 Mayo 2018 Fecha de publicación: 18 Junio 2018

\section{Resumen:}

Antecedentes: Los quistes y tumores odontogénicos (QTO) constituyen un grupo importante de las patologías orales. No existen datos epidemiológicos sobre la frecuencia de estas lesiones en la población nicaragüense. Objetivo: Establecer la frecuencia relativa de QTO en la población nicaragüense. Métodos: Se realizó un estudio retrospectivo en el que se analizaron los registros de biopsias excisionales del 2010 al 2016 de los departamentos de patología de los hospitales regionales de las ciudades de León, Managua, Estelí, Matagalpa y Jinotega. Se empleó la clasificación de la Organización Mundial de la Salud del 2017. El análisis fue descriptivo por medio de frecuencias relativas de las patologías estudiadas. Resultados: De un total de 13.102 reportes revisados se encontraron 109 casos $(0,8 \%)$. La distribución por sexo fue 50,9\% hombres y 49,9\% mujeres, principalmente en el intervalo de edad de 11 a 30 años. El quiste más frecuente fue el dentígero $(69,5 \%$ ) y el tumor odontogénico más frecuente fue el ameloblastoma (50 \%), principalmente en la mandíbula. Conclusión: Este estudio proporciona información epidemiológica sobre QTO en cinco ciudades de Nicaragua, la cual es esencial para identificar grupos de riesgo y posibles factores asociados, así como para diseñar sistemas de diagnóstico diferenciales y tratamiento adecuados.

Palabras clave: ameloblastoma, epidemiología oral, medicina oral, odontología, población nicaragüense, quiste dentígero, quistes odontogénicos, tumores odontogénicos.

\section{Abstract:}

Background: Odontogenic cysts and tumors (OCTs) are an important group of oral pathologies. There are no epidemiological data of the frequency of these lesions among Nicaraguans. Purpose: To establish the relative frequency of OCTs in Nicaraguan population. Methods: This was a retrospective study in which excisional biopsy records of regional hospital pathology departments from the cities of León, Managua, Estelí, Matagalpa, and Jinotega, obtained between 2010 and 2016, were analyzed. The pathologies were classified using the World Health Organization 2017 guidelines. Data analysis was descriptive through relative

Notas de autor:

a Autor de correspondencia. Correo electrónico: luis.quintana@fo.unanleon.edu.ni 
frequencies. Results: There were 109 OCT cases (0.8\%) out of 13,102 reports reviewed. Distribution by sex was $50.9 \%$ men and $49.9 \%$ women, mainly in the 11-to-30-year age range. The most frequent cyst was dentigerous cyst (69.5\%) and the most common odontogenic tumor was ameloblastoma (50\%), predominantly in the jaw. Conclusion: This study provides epidemiological information on OCTs from five Nicaraguan cities. These data are important to identify groups at risk and possible associated factors, and to implement differential diagnostic and adequate treatment systems.

Keywords: ameloblastoma, dentigerous cyst, dentistry, odontogenic cysts, odontogenic tumors, oral epidemiology, oral medicine, Nicaraguan population.

\section{Introducción}

Los quistes y tumores odontogénicos (QTO) constituyen un grupo importante de las patologías orales (1). Los quistes odontogénicos aparecen a cualquier edad, pero permanecen asintomáticos. Por lo tanto, no se les detecta temprano (1). En la mayoría de los casos, se identifican al obtener radiografías de rutina (figura 1). Su génesis está relacionada con la formación dental, pues el $90 \%$ de ellos se forma a partir de epitelio odontogénico o de sus restos embrionarios. Sin embargo, su etiología es aún desconocida en la mayoría de los casos $(2,3,4,5)$. Los tumores odontogénicos se relacionan con los quistes, que tienden a ser lesiones de mayor tamaño, pero también suelen pasar inadvertidos e identificarse durante exámenes radiográficos de rutina. Su tratamiento, la exéresis o extirpación quirúrgica son invasivos e incluso mutilantes y podría afectar la funcionalidad bucal (2). 


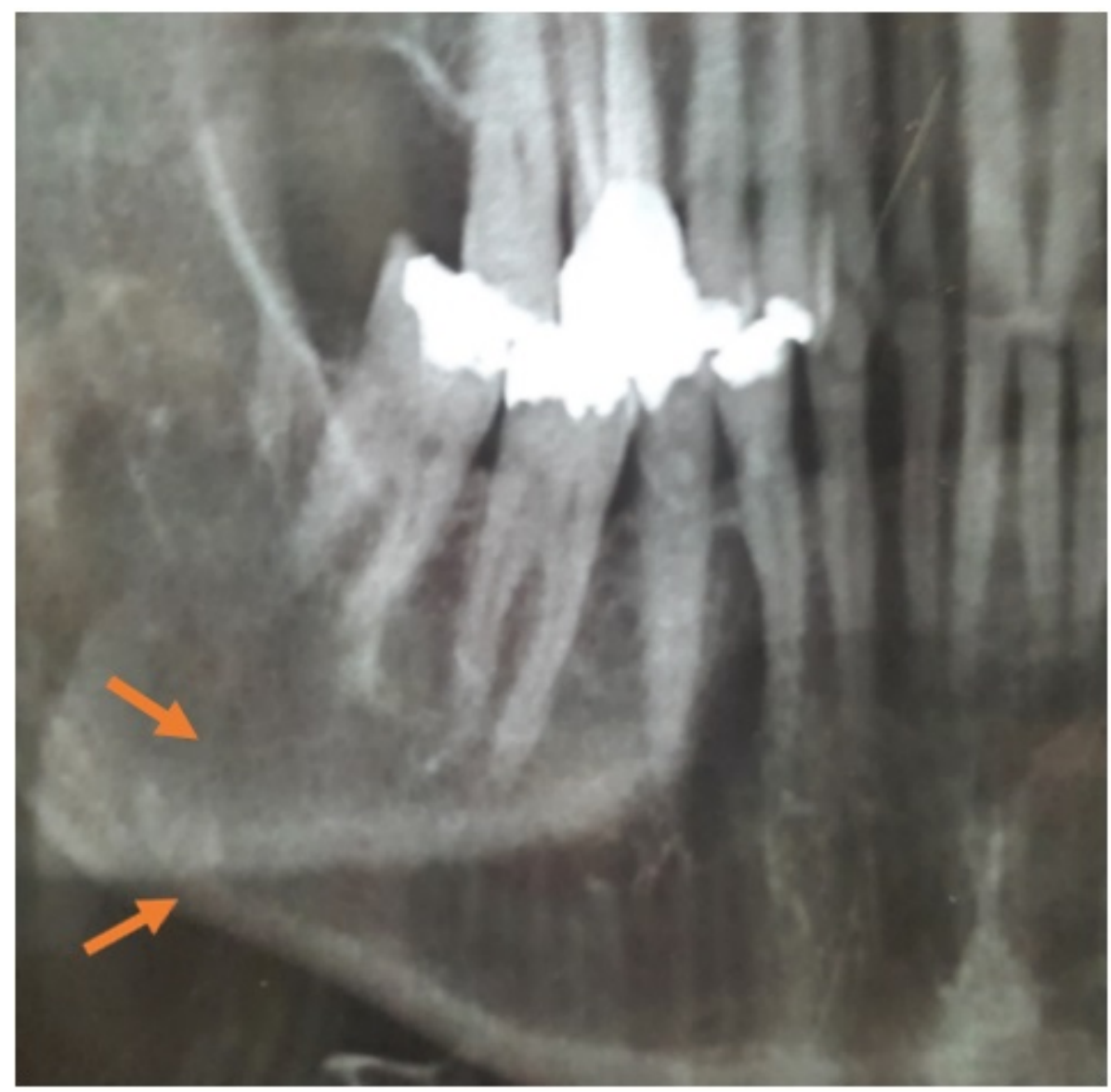

FIGURA 1

Ortopantomografía que muestra radiolucidez entre los premolares inferiores y correspondiente a un quiste o tumor de origen odontogénico Fuente: elaboración propia.

En comparación con otras entidades, los QTO son procesos patológicos poco frecuentes, cuya prevalencia oscila entre el 0,8 \% y el 4,7 \% de todas las lesiones de la región maxilofacial $(3,4)$. Conocer la epidemiología de los QTO es importante, porque permite establecer su expresión en diversas poblaciones, identificar los grupos de riesgo y reconocer los posibles factores asociados a su desarrollo, así como desarrollar sistemas de diagnóstico diferencial $(4,5)$. Con la identificación temprana de las características clínicas e imagenológicas de estas lesiones se contribuye a mejorar el pronóstico pues, a mayor tiempo de evolución, mayores son el daño a la persona y afectaciones como deformidades faciales, parestesias, pérdida dental e, incluso, muerte (1). Hay pocos estudios epidemiológicos de QTO en poblaciones latinoamericanas (2). Algunos reportes muestran una frecuencia del $4 \%$ en México (2), del 4,1 \% en Brasil (5) y del 12,1 \% en Uruguay (6). No se cuenta con estudios en Centroamérica. Con respecto a Nicaragua, existe un estudio de 1993 que utilizó la clasificación de las patologías de la Organización Mundial de la Salud (OMS), de 1992 (7). El objetivo de este estudio fue establecer la frecuencia de QTO en la población nicaragüense mediante la clasificación de la OMS del 2017.

\section{Materiales y métodos}

Este fue un estudio descriptivo retrospectivo que empleó información de los departamentos de patología de hospitales de referencia de Nicaragua: Hospital Escuela Óscar Danilo Rosales Argüello, en León (occidente del país); Hospital Dr. Roberto Calderón Gutiérrez y Hospital Militar Escuela Dr. Alejandro Dávila Bolaños, 
en Managua (región central del Pacífico); Hospital César Amador Molina, en Matagalpa; Hospital Escuela San Juan de Dios, en Estelí, y Hospital Victoria Motta, en Jinotega (estas últimas en el norte). Según datos del Instituto Nacional de Información del Desarrollo, en el 2012 Nicaragua tenía una población de 6,15 millones de habitantes. La población estimada en estas cinco ciudades fue de un poco más de 3 millones de habitantes (8).

El protocolo de la investigación recibió aprobación del Comité de Ética de la Universidad Nacional Autónoma de Nicaragua León. Se solicitó permiso a los directores del Sistema Local de Atención Integral de Salud en cada ciudad. Se seleccionaron 109 reportes que cumplían con los siguientes criterios de inclusión: datos completos, diagnósticos histopatológicos de QTO con base en la clasificación de la OMS del 2017 (9) y comprender el periodo 2010-2016. Se excluyeron reportes con datos incompletos y con diagnóstico histopatológico dudoso.

Los datos recolectados se registraron en fichas elaboradas para tal fin y se analizaron mediante el programa SPSS, versión 20, de IBM. Las variables analizadas fueron: diagnóstico de QTO, edad, sexo, hospital de donde procedía el caso, hueso afectado (maxilar/mandíbula). Los hallazgos se presentan mediante porcentajes.

\section{Resultados}

Se revisaron 13.102 reportes de biopsias del periodo de siete años escogido (enero del 2010-diciembre del 2016). Se encontraron 109 casos de QTO (0,8 \%). Un poco más de la mitad de estas lesiones resultaron ser quistes (54,1\%), y los demás, tumores (45,2\%). Los QTO en hombres se presentaron en una razón de 1:1,2. El quiste dentígero tuvo una frecuencia del $69,5 \%$, en tanto que el quiste radicular se presentó en el 20,3 $\%$ de los casos (tabla 1). El $50 \%$ de los tumores odontogénicos fueron ameloblastomas; el $18 \%$, mixomas odontogénicos, y el $16 \%$, tumores odontogénicos adenomatoides (tabla 2).

TABLA 1

Frecuencia de quistes de origen odontogénico según sexo

\begin{tabular}{lrrrrr}
\hline \multirow{2}{*}{ Tipo de quiste } & \multirow{2}{*}{ Total } & \multicolumn{4}{c}{ Sexo } \\
\cline { 2 - 7 } & & & Mujeres & \multicolumn{2}{c}{ Hombres } \\
\cline { 2 - 7 } & $\mathbf{n}(\%)$ & $\mathbf{n}$ & $(\%)$ & $\mathbf{n}$ & $(\%)$ \\
\hline Quistes gingivales de los lactantes & -- & - & - & - & - \\
\hline Quiste odontógeno ortoqueratinizado & $2(3,3)$ & 2 & $(7,1)$ & - & - \\
\hline Quiste dentígero & $41(69,5)$ & 16 & $(57,1)$ & 25 & $(80,6)$ \\
\hline Quiste periodontal lateral & -- & - & - & - & - \\
\hline Quiste gingival del adulto & -- & - & - & - & - \\
\hline Quiste odontógeno glandular & -- & - & - & - & - \\
\hline Quiste radicular & $12(20,3)$ & 9 & $(32,1)$ & 3 & $(9,7)$ \\
\hline Queratoquiste odontogénico & $4(6,7)$ & 1 & $(3,6)$ & 3 & $(9,7)$ \\
\hline Total & $59(100)$ & 28 & $(100)$ & 31 & $(100)$ \\
\hline
\end{tabular}


TABLA 2

Frecuencia de tumores de origen odontogénico según sexo

\begin{tabular}{lrrrrr}
\hline \multirow{2}{*}{ Tipo de tumor } & \multirow{2}{*}{ Total } & \multicolumn{3}{c}{ Sexo } \\
\cline { 2 - 7 } & & \multicolumn{2}{c}{ Mujeres } & \multicolumn{2}{c}{ Hombres } \\
\cline { 2 - 7 } & $\mathbf{n}(\%)$ & $\mathbf{n}$ & $(\%)$ & $\mathbf{n}$ & $(\%)$ \\
\hline Ameloblastoma & -- & - & - & - & - \\
\hline Tumor odontógeno escamoso & $2(5)$ & 2 & $(8)$ & - & - \\
\hline Tumor odontógeno epitelial calcificante & $8(16)$ & 1 & $(4)$ & 7 & $(24)$ \\
\hline Tumor odontógeno adenomatoide & -- & - & - & - & - \\
\hline Tumor odontógeno quístico calcificante & $1(2)$ & 1 & $(4)$ & - & - \\
\hline Fibroma ameloblástico & $1(2)$ & - & - & 1 & $(3)$ \\
\hline Fibrodontoma ameloblástico & $4(8)$ & 3 & $(13)$ & 1 & $(3)$ \\
\hline Odontoma & -- & - & - & - & - \\
\hline Odontoameloblastoma & -- & - & - & - & - \\
\hline Fibroma odontógeno & $9(18)$ & 4 & $(17)$ & 5 & $(17)$ \\
\hline Mixoma odontógeno & -- & - & - & - & - \\
\hline Cementoblastoma & $50(100)$ & 23 & $(100)$ & 28 & $(100)$ \\
\hline Total & & & & & \\
\hline
\end{tabular}

Fuente: elaboración propia.

El grupo etario con mayor frecuencia de quistes odontogénicos fue el de 21-30 años (73\%). En cuanto al quiste dentígero, el grupo etario menos afectado fue el de menores de 10 años (tabla 3). Por otra parte, los tumores odontogénicos fueron más frecuentes en el grupo de 11-20 años (38\%). Se halló una razón 2:1 para la afectación de la mandíbula con respecto al maxilar superior (tabla 4).

TABLA 3

Frecuencia de quistes odontogénicos según grupo etario

\begin{tabular}{|c|c|c|c|c|c|c|c|c|c|c|c|c|c|c|}
\hline \multirow{2}{*}{ Tipo de quiste } & \multicolumn{2}{|c|}{$<10$} & \multicolumn{2}{|c|}{$11-20$} & \multicolumn{2}{|c|}{$21-30$} & \multicolumn{2}{|c|}{$31-40$} & \multicolumn{2}{|c|}{$41-50$} & \multicolumn{2}{|c|}{$51-60$} & \multicolumn{2}{|c|}{$61>$} \\
\hline & $\mathbf{n}$ & $\%$ & $\mathrm{n}$ & $\%$ & $\mathrm{n}$ & $\%$ & $\mathrm{n}$ & $\%$ & $\mathbf{n}$ & $\%$ & $\mathrm{n}$ & $\%$ & $\mathbf{n}$ & $\%$ \\
\hline Quiste dentígero & 2 & 100 & 13 & 93 & 16 & 73 & 5 & 83 & 1 & 17 & 3 & 75 & 1 & 100 \\
\hline Quiste radicular & - & - & 1 & 7 & 6 & 27 & - & - & 5 & 83 & - & - & - & - \\
\hline $\begin{array}{l}\text { Queratoquiste } \\
\text { odontogénico }\end{array}$ & - & - & 2 & 11 & 2 & 25 & - & - & - & - & - & - & - & - \\
\hline $\begin{array}{l}\text { Quiste odontógeno } \\
\text { ortoqueratinizado }\end{array}$ & - & - & - & - & - & - & 1 & 17 & - & - & 1 & 25 & - & - \\
\hline Total & 2 & 100 & 16 & 100 & 24 & 100 & 6 & 100 & 6 & 100 & 4 & 100 & 1 & 100 \\
\hline
\end{tabular}


TABLA 4

Frecuencia de tumores de origen odontogénico según grupo etario

\begin{tabular}{|c|c|c|c|c|c|c|c|c|c|c|c|c|c|c|}
\hline \multirow{3}{*}{ Tipo de tumor } & \multicolumn{14}{|c|}{ Grupo etario } \\
\hline & \multicolumn{2}{|c|}{$<10$} & \multicolumn{2}{|c|}{$11-20$} & \multicolumn{2}{|c|}{$21-30$} & \multicolumn{2}{|c|}{$31-40$} & \multicolumn{2}{|c|}{$41-50$} & \multicolumn{2}{|c|}{$51-60$} & \multicolumn{2}{|c|}{$61>$} \\
\hline & n & (\%) & n & $(\%)$ & n & $(\%)$ & $\mathrm{n}$ & $(\%)$ & $\mathbf{n}$ & $(\%)$ & $\mathrm{n}$ & $(\%)$ & $\mathbf{n}$ & $(\%)$ \\
\hline Ameloblastoma & 1 & $(50)$ & 5 & $(31,2)$ & 3 & $(50)$ & 6 & $(75)$ & 6 & $(60)$ & 3 & $(60)$ & 1 & (33) \\
\hline $\begin{array}{l}\text { Tumor odontógeno } \\
\text { epitelial calcificante }\end{array}$ & - & - & - & - & - & - & - & - & 2 & $(20)$ & - & - & - & - \\
\hline $\begin{array}{l}\text { Tumor odontógeno } \\
\text { adenomatoide }\end{array}$ & - & - & 7 & $(43,7)$ & 1 & $(16$ & - & - & - & - & - & - & - & - \\
\hline $\begin{array}{l}\text { Fibroma } \\
\text { ameloblástico }\end{array}$ & - & - & 1 & $(6,3)$ & - & - & - & - & - & - & - & - & - & - \\
\hline $\begin{array}{l}\text { Fibrodontoma } \\
\text { ameloblástico }\end{array}$ & - & - & 1 & $(6,3)$ & - & - & - & - & - & - & - & - & - & - \\
\hline Odontoma & - & - & 2 & $(12,5)$ & - & - & - & - & - & - & 1 & (20) & 1 & (34) \\
\hline $\begin{array}{l}\text { Mixoma } \\
\text { odontógeno }\end{array}$ & 1 & $(50)$ & - & - & 2 & $(33,3)$ & 2 & (25) & 2 & (20) & 1 & (20) & 1 & (33) \\
\hline Total & 2 & (100) & 16 & $(100)$ & 6 & $(100)$ & 8 & (100) & 10 & $(100)$ & 5 & (100) & 3 & $(100)$ \\
\hline
\end{tabular}

Fuente: elaboración propia.

\section{Discusión}

En el presente estudio se encontraron 109 casos con diagnósticos de QTO en el periodo enero del 2010 y diciembre del 2016, en las ciudades nicaragüenses de León, Managua, Matagalpa, Jinotega y Estelí. La prevalencia total fue del 0,8 \%. En orden de frecuencia, los quistes más frecuentes fueron el dentígero, el periapical y el queratoquiste odontogénico. Por su parte, los tumores odontogénicos más comunes fueron el ameloblastoma, el tumor odontogénico adenomatoide y el mixoma odontogénico. En la población nicaragüense estudiada, los QTO no presentaron predilección por sexo, aunque fueron más comunes en pacientes jóvenes (11-30 años de edad).

Con respecto a la prevalencia general de los QTO, cabe señalar que son lesiones raras y que, a pesar de la dificultades diagnósticas que pueden existir en los servicios de salud del país, la cifra fue similar a lo que aparece reportado en la literatura (0,8 \%-4,5\%) (3). Entre los países latinoamericanos, las tasas reportadas de estas lesiones incluyen: México, con un $4 \%$ (4), y Brasil, con un 4,1 \% (5). En Uruguay, los quistes se presentan en un $12,1 \%$ (6). Estas cifras son elevadas. En cambio, en otros países como Estados Unidos (10), India (11), Turquía (12) y Libia (13), estas lesiones se presentan en menos del 1,5\% de la población.

En orden de frecuencia, el quiste más común fue el dentígero, seguido del radicular y el queratoquiste odontogénico. Este hallazgo probablemente se deba a que en el sistema de salud de Nicaragua se realiza regularmente el estudio histopatológico de las lesiones periapicales de dientes extraídos. Esta distribución de la morbilidad de los quistes podría diferente, pues otros autores como Neville y colaboradores (14), Cawson y colaboradores (15) y Villasis-Sarmiento y colaboradores (2) han reportado el quiste radicular como el más frecuente. En el caso del queratoquiste odontogénico, cabe señalar que en el 2017 la OMS lo reclasificó como un quiste, pues antes se catalogaba como un tumor verdadero (9). En los reportes revisados hubo variaciones en el criterio de diagnóstico para clasificar dicha condición como quiste o tumor, todas relacionadas con el año en que se recolectaron datos de morbilidad. Por esta razón, es difícil efectuar análisis comparados con estudios anteriores al 2017.

En este estudio no hubo predilección en cuanto al sexo. Otros autores como Castillo y Dávila (7), Neville y colaboradores (14), Raj y colaboradores (11), Cawson y colaboradores (15) y Cecotti y colaboradores (16) 
identificaron una ligera tendencia de aparición hacia el sexo masculino. Estas variaciones deberían analizarse en relación con los factores de riesgo y los determinantes sociales en cada población.

Los tumores más encontrados en este estudio fueron ameloblastoma, mixoma odontogénico y odontogénico adenomatoide. El ameloblastoma es el tumor odontogénico más común en países como México, Brasil, Venezuela, Egipto y Turquía. Autores como Neville y colaboradores (14), Contreras y colaboradores (3), Mosqueda-Taylor y colaboradores (4), Lima-Verne-Osterne y colaboradores (5) lo describen como el tumor odontogénico más frecuente que afecta principalmente el grupo etario de 11-20 años, aunque la media de edad varía entre países. Por ejemplo, Reichart y colaboradores (17) informaron una edad media de 36 años, mientras que Ledesma-Montes y colaboradores (18) describieron una edad media de ocurrencia a los 41,4 años. Cabe señalar que para varios autores el odontoma es uno de los tumores más frecuentes y aparece de la primera a tercera décadas de vida $(5,6,7)$. En este estudio de población nicaragüense los QTO se presentaron mayoritariamente en personas jóvenes y en afectación similar a los sexos masculino y femenino.

Una de las fortalezas de este estudio fue el número de reportes revisados y que los datos se obtuvieron de hospitales de referencia. Otra fortaleza fue haber realizado el corte donde se definieron los grupos mayormente afectados por estas patologías. Entre las limitantes del estudio está la clasificación de los QTO que se usó en los reportes, pues fueron anteriores a la reclasificación del 2017; en el sistema de salud de Nicaragua no existen criterios uniformes para clasificar los QTO en todos los hospitales, pues están al criterio de cada patólogo y, por lo general, la clasificación de la OMS del 2017 es poco conocida. Aunque el diseño empleado permite describir la frecuencia de estas patologías en la población, se requiere más investigación. Un estudio analítico posterior deberá identificar los factores de riesgo para QTO en la población joven.

\section{Conclusiones}

Este estudio proporciona información epidemiológica sobre quistes y tumores odontogénicos en población nicaragüense.

Los quistes y tumores odontogénicos se presentan en más alta frecuencia a edades tempranas, sin diferencia en cuanto al sexo.

\section{Recomendaciones}

Estudios correlacionales posteriores deberían identificar los factores de riesgo y determinantes sociales asociados a los QTO en los diferentes grupos etarios analizados.

\section{Referencias}

1. García-Rubio A, Bujaldón-Daza AL, Rodríguez-Archilla A. Lesiones periapicales: Diagnóstico y tratamiento. Av Odontoestomatol. 2015; 31(1): 31-42. http://dx.doi.org/10.4321/S0213-12852015000100005

2. Villasis-Sarmiento L, Portilla-Robertson J, Meléndez-Ocampo A, Gaitán-Cepeda LA, Leyva-Huerta ER. Prevalence and distribution of odontogenic cysts in a Mexican sample: A 753 cases study. J Clin Exp Dent. 2017; 9(4): e531e538. http://dx.doi.org/10.4317/jced.53627

3. Contreras D, Dellán A, Granadillo K, Tinoco P, Salazar N, Villarroel-Dorrego M. Estudio clínico transversal de tumores odontogénicos: análisis clínico patológico de 450 casos. Acta Bioclínica [internet]. 2013; 3(5): 98-115. Disponible en: http://erevistas.saber.ula.ve/index.php/actabioclinica/article/view/4405

4. Mosqueda-Taylor A, Meneses-García A, Ruiz-Godoy-Rivera LM, Suárez-Roa ML, Luna-Ortiz K. Tumores odontogénicos malignos: Estudio retrospectivo y colaborativo de 7 casos. Med Oral. 2003; 8: 110-21. 
5. Lima-Verde-Osterne R, Turatti E, Cordeiro-Teixeira R, Barroso-Cavalcante R. The relative frequency of odontogenic tumors: A study of 376 cases in a Brazilian population. Med Oral Patol Oral Cir Bucal. 2017; 22(2): e193. https://doi.org/10.1034/j.1600-0714.2002.00133.x

6. Fungi Monetti M. Revisión analítica de los quistes odontogénicos: Archivo de la Cátedra de Anatomía Patológica Facultad de Odontología UdelaR. Odontoestomatol. 2011; 13(18): 56-65.

7. Castillo BA, Dávila EM. Frecuencia de quistes y tumores de origen odontogénico en los Hospitales "Oscar Danilo Rosales”. León. y “Manolo Morales”. Managua. 1980-1988. León, Nicaragua: Universidad Nacional Autónoma de Nicaragua León; 1993.

8. Instituto Nacional de Información de Desarrollo (INIDE). Anuario estadístico 2012 [internet]. Managua: Nicaragua: INIDA; 2012. Dosponible en: http://www.inide.gob.ni/estadisticas/Cifras\%20municipales\%20a\% C3\%B1o\%202012\%20INIDE.pdf

9. Philipsen HP, Reichard PA. Classification of odontogenic tumours. A historical review. J Oral Pathol Med. 2006; 35(9): 525-9. https://doi.org/10.1111/j.1600-0714.2006.00470.x

10. Buchner A, Merrell PW, Carpenter WM. Relative frequency of central odontogenic tumors: a study of 1,088 cases from Northern California and comparison to studies from other parts of the world. J Oral maxillofac Surg. 2006; 64(9): 1343-52. https://doi.org/10.1016/j.joms.2006.05.019

11. Raj A, Ramesh G, Nagarajappa R, Pandey A, Raj A. Prevalence of odontogenic lesions among the Kanpur population: an institutional study. J Exp Ther Oncol. 2017; 12(1): 35-42.

12. Sekerci AE, Nazlım S, Etoz M, Denız K, Yasa Y. Odontogenic tumors: a collaborative study of 218 cases diagnosed over 12 years and comprehensive review of the literature. Med Oral Patol Oral Cir Bucal. 2015; 20(1): e34-44. http://dx.doi.org/doi:10.4317/medoral.19157

Goteti SH. Odontogenic tumors: A review of 675 cases in Eastern Libya. Niger J Surg. 2016; 22(1): 37-40. https:// doi.org/10.4103/1117-6806.176397

14. Neville BW, Damm DD, Chi AC, Allen CM. Oral and maxillofacial pathology. St Louis, Missouri: Elsevier; 2015.

15. Cawson RA, Cawson E, Odell E. Cawson fundamentos de medicina y patología oral: Barcelona, España: Elsevier; 2009.

16. Ceccotti S, Forteza C, Luberti F. El diagnóstico en clínica estomatológica. Buenos Aires, Argentina: Panamericana; 2007.

17. Reichart P, Philipsen H, Sonner S. Ameloblastoma: biological profile of 3677 cases. Europ J Cancer B Oral Oncol. 1995; 31B(2): 86-99. https://doi.org/10.1016/0964-1955(94)00037-5

18. Ledesma-Montes C, Mosqueda-Taylor A, Carlos-Bregni R, De León ER, Palma-Guzmán J, Páez-Valencia C, Meneses-García A. Ameloblastomas: a regional Latin-American multicentric study. Oral Dis. 2007; 13(3):303-7. https://doi.org/10.1111/j.1601-0825.2006.01284.x

\section{Notas}

* Artículo de investigación.

\section{Licencia Creative Commons CC BY 4.0}

Cómo citar este artículo: Quintana Salgado LA, Espinoza Hernández LJ, Rodríguez Vílchez JJ, Guerrero Midence CD, Rugama Díaz, HJ. Frecuencia de quistes y tumores odontogénicos en población nicaragüense. Estudio retrospectivo a siete años. Univ Odontol. 2018 ene-jun; 37(78). https://doi.org/10.11144/Javeria na.uo37-78.fqto 